Article

\title{
Octadecylamine-Grafted Graphene Oxide Helps the Dispersion of Carbon Nanotubes in Ethylene Vinyl Acetate
}

\author{
Li-Chuan Jia ${ }^{1}$, Zhong-Han Jiao ${ }^{1}$, Ding-Xiang Yan ${ }^{2, *}$ and Zhong-Ming $\mathrm{Li}^{1, *}$ \\ 1 College of Polymer Science and Engineering, State Key Laboratory of Polymer Materials Engineering, \\ Sichuan University, Chengdu 610065, China; jialichuan@stu.scu.edu.cn (L.-C.J.); \\ jiaozhonghan@stu.scu.edu.cn (Z.-H.J.) \\ 2 School of Aeronautics and Astronautics, Sichuan University, Chengdu 610065, China \\ * Correspondence: yandingxiang@scu.edu.cn (D.-X.Y.); zmli@scu.edu.cn (Z.-M.L.); \\ Tel.: +86-028-8540-0211 (D.-X.Y. \& Z.-M.L.)
}

Received: 11 August 2017; Accepted: 25 August 2017; Published: 27 August 2017

\begin{abstract}
In this paper, the dispersion of carbon nanotube (CNT) in ethylene vinyl acetate (EVA) is demonstrated to be significantly improved by the addition of octadecylamine (ODA)-grafted graphene oxide (GO) (GO-ODA). Compared to the CNT/EVA composite, the resultant GO-ODA/CNT/EVA (G-CNT/EVA) composite shows simultaneous increases in tensile strength, Young's modulus and elongation at break. Notably, the elongation at break of the G-CNT/EVA composite still maintains a relatively high value of $1268 \%$ at $2.0 \mathrm{wt} \% \mathrm{CNT}$ content, which is more than 1.6 times higher than that of CNT/EVA composite (783\%). This should be attributed to the homogeneous dispersion of CNT as well as the strong interfacial interaction between CNT and EVA originating from the solubilization effect of GO-ODA. Additionally, the G-CNT/EVA composites exhibit superior electrical conductivity at low CNT contents but inferior value at high CNT contents, compared to that for the CNT/EVA composite, which depends on the balance of CNT dispersion and the preservation of insulating GO-ODA. Our strategy provides a new pathway to prepare high performance polymer composites with well-dispersed CNT.
\end{abstract}

Keywords: carbon nanotube; homogeneous dispersion; ethylene vinyl acetate; mechanical performance; electrical conductivity

\section{Introduction}

Carbon nanotube (CNT) has been regarded as a very promising nanofiller for polymer matrices to achieve high performance and multifunction, due to its extraordinary mechanical, electrical, and thermal properties [1-8]. The CNT-based polymer composites are expected to make enormous technological and commercial impacts in view of extensive applications in sensing devices $[9,10]$, electrical shielding and heating devices [11-15], as well as advanced composites for space and aircrafts $[16,17]$. Nevertheless, the resultant performances show much lower efficiency than expected though significant efforts have been made. This is mainly attributed to the fact that CNTs easily agglomerate in polymer matrices because of the high aspect ratio and strong van der Waals interactions, which would greatly limit their utilization $[18,19]$.

Recently, there are growing efforts on controlled surface modification of CNT to realize well dispersion through covalent or noncovalent approaches [20-26]. The covalent functionalization lacks economic benefits and would introduce many defects that inevitably deteriorate the intrinsic performances of CNT, whereas the noncovalent functionalization is particularly attractive because it preserves nearly all intrinsic features of CNT. For traditional noncovalent approaches, homogeneous 
CNT dispersion was always facilitated by surfactant and polymer wrapping based on van der Waals interactions or $\pi-\pi$ stacking interactions. However, the surfactants may largely affect the properties of the obtained composites and the available polymers for CNT solubilization are limited [27,28]. Recently, graphene oxide (GO) was demonstrated as a high-efficient surfactant to disperse CNT due to the high solubility and adhesion of CNT onto the flat GO sheets through strong $\pi-\pi$ stacking interaction [29-33]. For instance, Liao et al. reported that the incorporation of GO prominently improved the dispersion of CNTs in poly(vinyl alcohol) (PVA) and the resulted GO-CNT/PVA composite showed great improvements in the yield strength and Young's modulus compared to those of CNT/PVA composite [31]. Fu et al. demonstrated that the tribological performance of CNT/epoxy composite was significantly enhanced by the incorporation of GO that improves the dispersion of CNT [32]. Although significant progress was achieved in developing high-performance materials, it should be noted that the GO-dispersed CNT was mainly applied in the strong polar polymer matrices, such as PVA, epoxy, and polyurethane, owing to the presence of hydrophilic functional groups (hydroxyl, epoxide, and carboxylic groups). The highly hydrophilic feature of GO makes it hardly achieve the favorable dispersion quality of CNT in weak or non-polar polymer matrices, which greatly restricts the development of such type of composites for advanced functional materials. Recently, tremendous attentions have been paid to the oleophylic modification of GO by grafting long alkyl chains to improve its dispersion in non-polar organic solvents and enhances its compatibility with weak or non-polar polymer matrices [34-36]. Inspired by the high efficiency of GO to disperse $\mathrm{CNT}$ in strong polar polymers, it is reasonable to hypothesize that long alkyl chain grafted GO should be promising and ideal to realize the efficient dispersion of CNT in weak or non-polar polymers.

In the current study, octadecylamine (ODA) grafted GO (GO-ODA) was synthesized to assist the dispersion of CNT in ethylene vinyl acetate (EVA), because CNT-based EVA composites are widely used for various applications such as packaging films, adhesives, antistatic field and electromagnetic shielding in view of their excellent flexibility, durability, and chemical resistance [37-39]. Significantly improved dispersion of CNT was achieved because of the strong solubilization of GO-ODA to CNT. The effect of CNT dispersion on the mechanical and electrical properties of EVA composites was investigated thoroughly. This solubilization approach for CNT with the assistance of GO-ODA is confirmed to be an effective strategy to fabricate high-performance CNT-based polymer composites.

\section{Materials and Methods}

\subsection{Materials}

The commercially available EVA containing $28 \mathrm{wt} \%$ of vinyl acetate was kindly provided by Beijing Dongfang Petroleum Chemical Co. (Beijing, China). The density is $0.92 \mathrm{~g} / \mathrm{cm}^{3}$ and the melt flow rate is $25 \mathrm{~g} /(10 \mathrm{~min})\left(190^{\circ} \mathrm{C}, 21.6 \mathrm{~N}\right)$. Graphene oxide $(\mathrm{GO})$ was prepared from expanded graphite, which was provided by Qingdao Haida Graphite Co., Ltd. (Qingdao, China) with an expansion rate of $200 \mathrm{~mL} / \mathrm{g}$, by the modified Hummers method, as described in our previous work [40]. CNT (NC 7000 series), with average diameter of $9.5 \mathrm{~nm}$, length $1.5 \mu \mathrm{m}$, surface area $250-300 \mathrm{~m}^{2} / \mathrm{g}$, and $90 \%$ carbon purity, was supplied by Nanocyl S.A., (Sambreville, Belgium). ODA, xylene, deionized water and anhydrous ethanol were supplied by Chengdu Kelong Chemical Reagent Factory, Chengdu, China. All these chemicals were analytical reagent and used without further purification.

\subsection{Preparation of GO-ODA}

GO-ODA was prepared by facile refluxing of GO and ODA. Specifically, $0.6 \mathrm{~g}$ GO was initially dispersed in $300 \mathrm{~mL}$ deionized water via vigorous agitation and ultrasonic treatment for $60 \mathrm{~min}$. Then the resulting uniform suspension was mixed with ODA/ethanol solution $(0.9 \mathrm{~g}$ in $90 \mathrm{~mL})$ in a three-neck flask. The mixture was refluxed with intensified mechanical stirring for $24 \mathrm{~h}$ at $85^{\circ} \mathrm{C}$ and filtrated by a PTFE membrane ( $0.2 \mu \mathrm{m}$ pore size). The filtrated powder was then washed thoroughly 
with ethanol and filtered to remove the remaining ODA. Finally, the mixture was dried in a vacuum oven overnight at $60^{\circ} \mathrm{C}$ for $48 \mathrm{~h}$.

\subsection{Preparation of GO-ODA/CNT/EVA Composites}

The schematic representation for the preparation of GO-ODA/CNT/EVA composites is illustrated in Figure 1. Firstly, GO-ODA was suspended in xylene via ultrasonication for $30 \mathrm{~min}$, then CNT was added to GO-ODA dispersion (1:1 weight ratio) and further ultrasonicated for $60 \mathrm{~min}$. Meanwhile, EVA was completely dissolved in hot xylene $\left(75^{\circ} \mathrm{C}\right)$ by mechanical stirring for $60 \mathrm{~min}$. Subsequently, GO-ODA/CNT/xylene dispersion was poured into the EVA/xylene solution and the mixture was continuously stirred for $30 \mathrm{~min}$ at $75{ }^{\circ} \mathrm{C}$. Afterwards, the mixture was recovered by flocculation with ethanol, followed by filtering and drying in a vacuum oven $\left(60^{\circ} \mathrm{C}\right)$ for $48 \mathrm{~h}$. Finally, the dried mixture was compression molded under the pressure of $10 \mathrm{MPa}$ at $160{ }^{\circ} \mathrm{C}$, after preheating for $5 \mathrm{~min}$. The resultant GO-ODA/CNT/EVA composites were marked as G-CNT/EVA composites. For comparison, the CNT/EVA composites were also prepared under the same processing conditions.

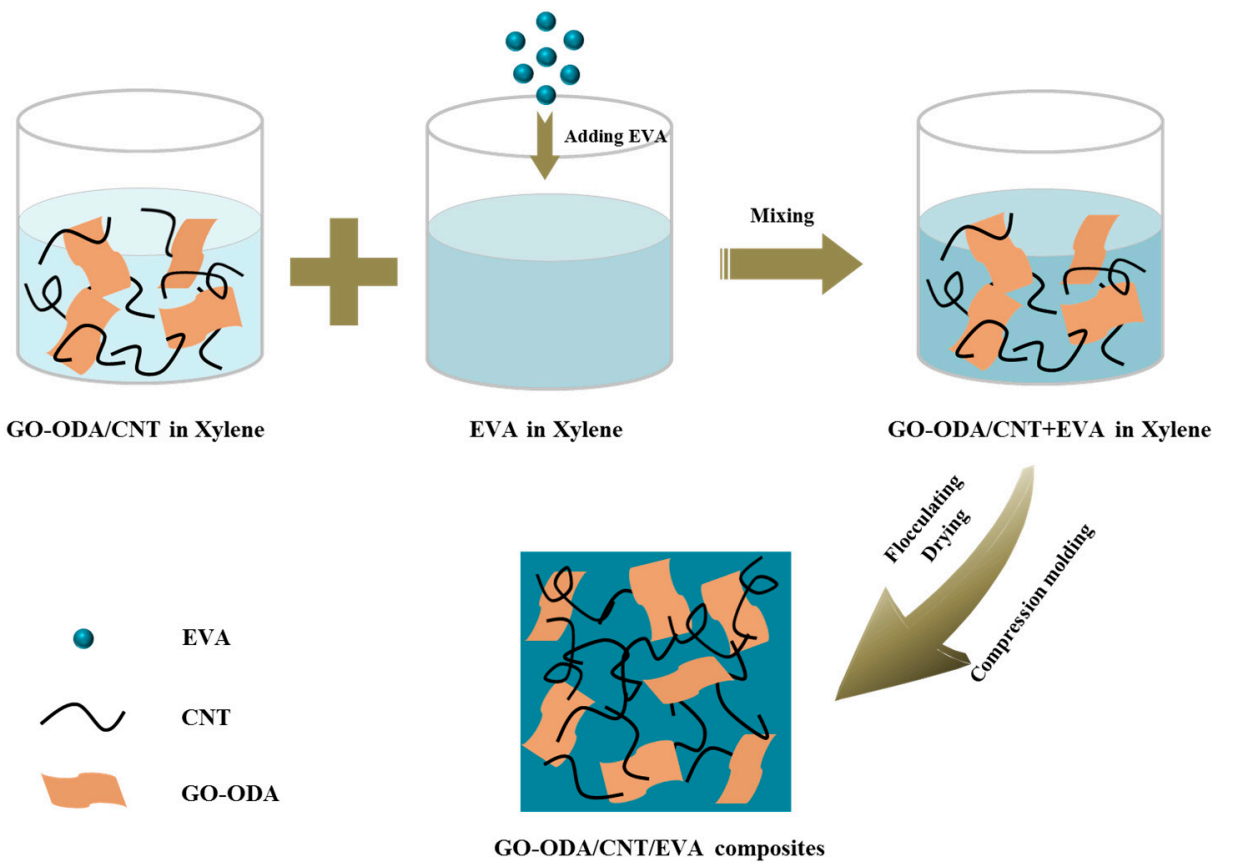

Figure 1. Scheme of the fabrication process of the G-CNT/EVA composites.

\subsection{Characterizations}

Fourier-transform infrared spectroscopy (FTIR) spectra were recorded with a $2 \mathrm{~cm}^{-1}$ spectral resolution on a Nicolet 6700 FTIR spectrometer (Thermo Nicolet, Waltham, MA, USA) in transmission mode. GO or GO-ODA was ground with $\mathrm{KBr}$ and pressed into $\mathrm{KBr}$ disks for FTIR measurements. X-ray diffraction (XRD) data was collected with a DX-1000 diffractometer (Dandong Fangyuan Instrument Co., Ltd., Liaoning, China) using CuKa irradiation at $40 \mathrm{kV}$ in a scanning range from $2^{\circ}$ to $30^{\circ}$. Water contact angle was measured by using a DSA 30 KRUSS drop shape analyzer (Kruss, Hamburg, Germany). Thermogravimetric analysis (TGA) was carried out to evaluate the thermal stability of GO and GO-ODA on a NETZSCH 209F1 (Netzsch, Bavarian, Germany), at a heating rate of $10^{\circ} \mathrm{C} / \mathrm{min}$ over $40-600{ }^{\circ} \mathrm{C}$ under nitrogen atmosphere. Scanning electron microscopy (SEM) images were taken by a field emission SEM (Inspect-F, FEI, Hillsboro, OR, USA) at an accelerating voltage of $20 \mathrm{kV}$. The specimens were cryo-fractured in liquid nitrogen and the fracture surfaces were coated with a thin layer of gold before morphological observation. The volume conductivity of the composite above $10^{-6} \mathrm{~S} / \mathrm{m}$ was carried out on a four-point probe instrument (RTS-8, Guangzhou Four-Point 
Probe Technology Co., Ltd., Guangzhou, China) and the volume conductivity below $10^{-6} \mathrm{~S} / \mathrm{m}$ was measured with a Keithley electrometer Model 4200-SCS (Keithley, Beaverton, OR, USA). Tensile test was performed on an Instron universal test instrument (Model 5576, Instron Instruments, Norwood, MA, USA) at room temperature. The loading rate was $100 \mathrm{~mm} / \mathrm{min}$ and the gauge length was $20 \mathrm{~mm}$. More than five samples were tested to calculate the average value and standard deviations.

\section{Results}

\subsection{Characterization of $G O-O D A$}

FTIR spectra were performed to determine the chemical changes that occurred during the refluxing of ODA and GO. As shown in Figure 2a, the absorption bands of GO appear at 1710, 1642, 1420, and $1064 \mathrm{~cm}^{-1}$, corresponding to $\mathrm{C}=\mathrm{O}$ in carboxyl group, $\mathrm{C}=\mathrm{C}$ in aromatic ring, $\mathrm{C}-\mathrm{OH}$ stretching and $\mathrm{C}-\mathrm{O}-\mathrm{C}$ in epoxide, respectively [36]. The broad peak appearing at $3256 \mathrm{~cm}^{-1}$ is assigned to the hydroxyl groups. When it comes to GO-ODA, the existence of the octadecyl chain is clearly demonstrated because of the emerging peaks at $2919 \mathrm{~cm}^{-1}$ and $2850 \mathrm{~cm}^{-1}\left(-\mathrm{CH}_{2}\right.$ stretching in the octadecyl chain) as well as the peak at $720 \mathrm{~cm}^{-1}$ [41,42]. Furthermore, the weakened intensity of $\mathrm{C}-\mathrm{O}-\mathrm{C}$ and disappearance of $\mathrm{C}=\mathrm{O}$ in carboxyl group indicate the amidation reaction between the amine functionality of ODA with the epoxy and carboxyl functionality of GO, confirming the nucleophilic substitution between ODA with GO occurred during the refluxing [36]. The new peaks at $1576 \mathrm{~cm}^{-1}$ (N-H bending of amide) and $1470 \mathrm{~cm}^{-1}$ (C-N stretch of amide) in GO-ODA also indicate the presence of amide-carbonyl bond between ODA molecule and GO. All these results affirm the intercalation and chemical reaction of ODA with GO, which is in line with the reported in the literature [36,43]. The XRD curves of GO and GO-ODA are presented in Figure 2b. Compared to diffraction peak of GO at $9.6^{\circ}$, the peak for GO-ODA shifts to smaller angles of $5.3^{\circ}$, corresponding to the increase in the intra-gallery spacing from $0.89 \mathrm{~nm}$ to $1.7 \mathrm{~nm}$. The enlarged intra-gallery space indicates the intercalation of the octadecyl chains between the GO nanosheets, supporting the reaction of ODA and GO. The influence of functionalization on the wetting property of GO was confirmed by the contact angle measurements. The contact angle of GO-ODA increases to $110.4^{\circ}$ in comparison with that of hydrophilic $\mathrm{GO}\left(73.2^{\circ}\right)$, revealing that the attached hydrophobic hydrocarbon chains make the GO-ODA hydrophobic (Figure 2c). TGA was further used to analyze the amination of ODA on GO surface and calculate the grafting ratio of ODA. As shown in Figure 2d, ODA shows a rapid weight loss starting at a low temperature $\left(120^{\circ} \mathrm{C}\right)$, and is almost exhausted when the temperature reaches $300^{\circ} \mathrm{C}$. In case of GO, two significant weight loss stages are observed, corresponding to the volatilization of water between 40 and $100^{\circ} \mathrm{C}$ and the pyrolysis of the labile oxygen containing functional groups on GO between 170 and $270{ }^{\circ} \mathrm{C}$, respectively. Compared to GO and ODA, the larger weight loss of GO-ODA in the range of $200-500{ }^{\circ} \mathrm{C}$ is mainly attributed to the decomposition of covalently bonded ODA on GO surface, demonstrating the successful graft modification of GO with ODA. The grafting ratio of ODA is calculated to about $9.1 \mathrm{wt} \%$ according to the yields of residual carbon at $600{ }^{\circ} \mathrm{C}$ [36]. 


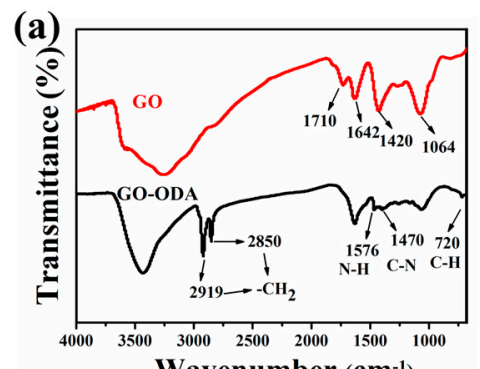

(c)
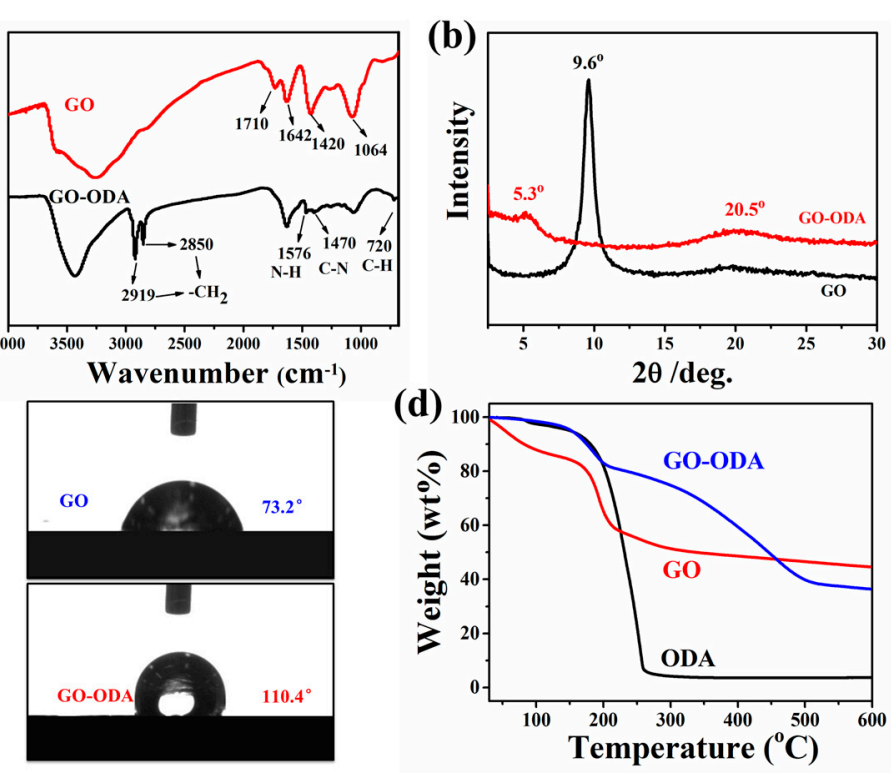

Figure 2. (a) Fourier-transform infrared spectroscopy (FTIR) spectra of graphene oxide (GO) and octadecylamine-grafted GO (GO-ODA); (b) X-ray diffraction (XRD) patterns of GO and GO-ODA; (c) Surface water contact angle of GO and GO-ODA; (d) TGA curves of ODA, GO and GO-ODA.

\subsection{Dispersion of CNT in EVA}

The dispersion of nanofillers has a direct correlation with the mechanical, electrical, and other properties of nanofiller/polymer composites. The fractured surface of the CNT-based EVA composite was observed with SEM to assess the CNT dispersion, as shown in Figure 3. For the G-CNT/EVA composites, CNTs are uniformly dispersed in EVA matrix without any visible aggregations (Figure 3a,b). While for the CNT/EVA composites, CNT agglomerations (labeled by red circle) are obviously observed (Figure 3c,d), mainly due to the intrinsic strong Van der Waals force of CNTs. These results indicate that GO-ODA plays a role of compatilizer for CNTs and EVA, which facilitates the dispersion of CNTs. Moreover, compared with the smooth fractured surface of $2.0 \mathrm{wt} \%$ CNT/EVA composite, the $2.0 \mathrm{wt} \% \mathrm{G}-\mathrm{CNT} / \mathrm{EVA}$ composite shows a relatively rough fractured surface, demonstrating the excellent compatibility and strong interfacial adhesion of CNTs with EVA [36]. Based on the aforementioned analysis, the application of GO-ODA not only improves the CNT dispersion in EVA matrix but also enhances their interface adhesion, which should be attributed to the $\pi-\pi$ stacking interaction between CNTs and GO-ODA and the interpenetration of GO-ODA chains with EVA chains. The well-dispersed CNTs in EVA matrix and high compatibility of CNTs with EVA are expected to impart the G-CNT/EVA composites with superior mechanical and electrical properties. 

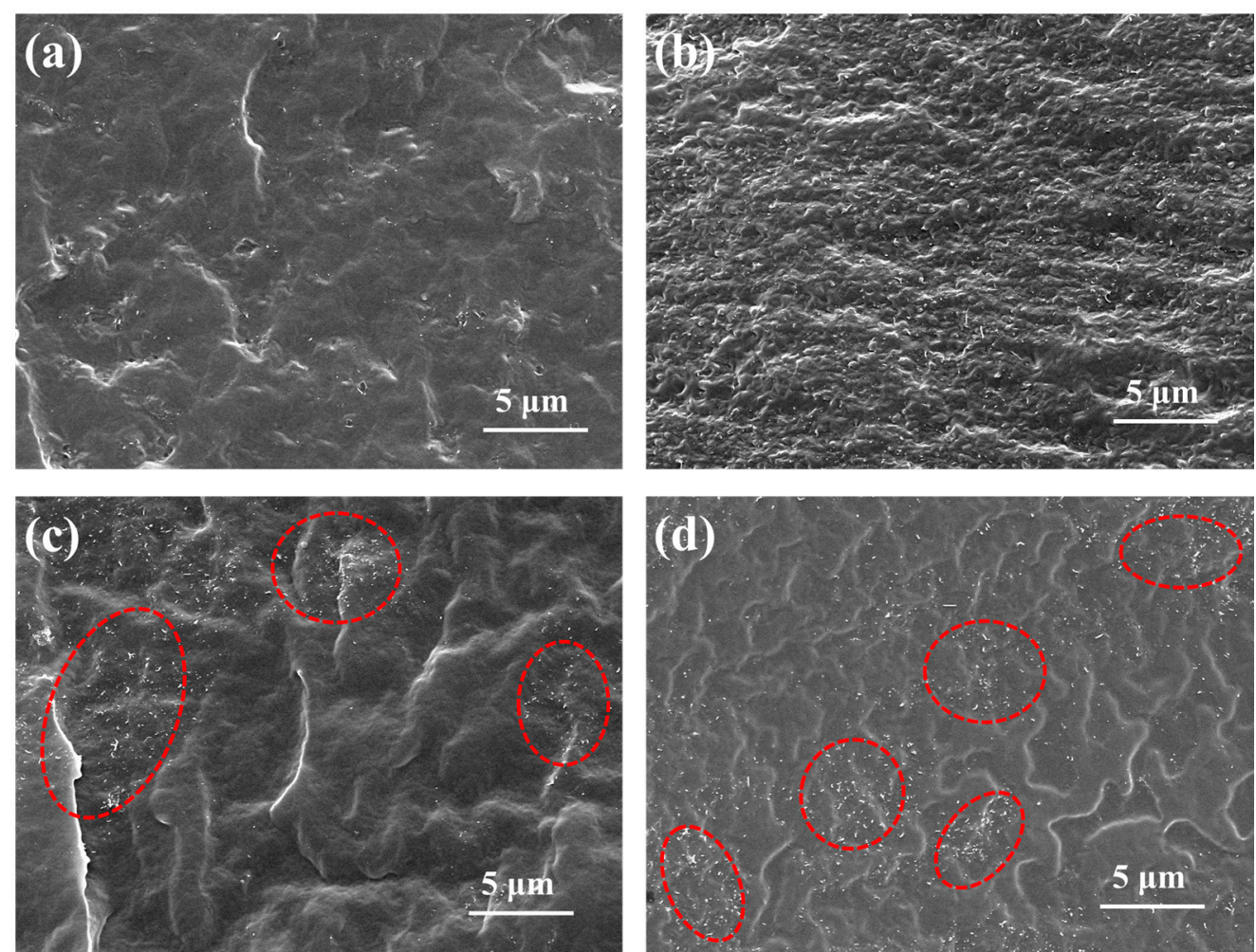

Figure 3. SEM images of the G-CNT/EVA composites with (a) $0.3 \mathrm{wt} \%$ and (b) $2.0 \mathrm{wt} \% \mathrm{CNT}$, respectively. SEM images of the CNT/EVA composites with (c) $0.3 \mathrm{wt} \%$ and (d) $2.0 \mathrm{wt} \% \mathrm{CNT}$, respectively. The red circles in (c) and (d) refer to the CNT agglomerations.

\subsection{Effects of CNT Dispersion on Mechanical Properties}

Figure 4a shows typical stress-strain curves of pure EVA, G-CNT/EVA, and CNT/EVA composites. With CNT incorporation, both G-CNT/EVA and CNT/EVA composites show increased tensile strength and Young's modulus, but decreased elongation at break, compared to those for pure EVA. The average Young's modulus, tensile strength, and elongation at break are calculated and illustrated in Figure $4 b-d$, respectively. The tensile strength and Young's modulus of the $0.3 \mathrm{wt} \%$ CNT/EVA composite are 22.7 and $13.5 \mathrm{MPa}$, which correspond to $56.6 \%$ and $53.4 \%$ increases from 14.5 and $8.8 \mathrm{MPa}$ for pure EVA. Further increasing CNT content to $2.0 \mathrm{wt} \%$ in the CNT/EVA composite, the Young's modulus increases to $15.5 \mathrm{MPa}$ due to the high stiffness of CNT, while the tensile strength drops to $15.3 \mathrm{MPa}$, mainly originating from the reduced elongation at break, which should be attributed to the heavy agglomeration of CNT in EVA matrix. This phenomenon is in line with previously reported CNT reinforced polymer composites $[7,25]$. When it comes to G-CNT/EVA composites, the improved CNT dispersion significantly boosts up the elongation at break as compared to the CNT/EVA composite. For instance, a more than $61.9 \%$ increase in elongation at break from $783 \%$ to $1268 \%$ is achieved at $2.0 \mathrm{wt} \% \mathrm{CNT}$ content. Moreover, the G-CNT/EVA composites exhibit much higher tensile strength and Young's modulus than those of the CNT/EVA composites. The excellent mechanical performance of the G-CNT/EVA composites should be attributed to the improved dispersion of CNT and the high compatibility between CNTs and EVA molecular chain with the assist of GO-ODA, both of which facilitate effective stress transfer in the composites. 

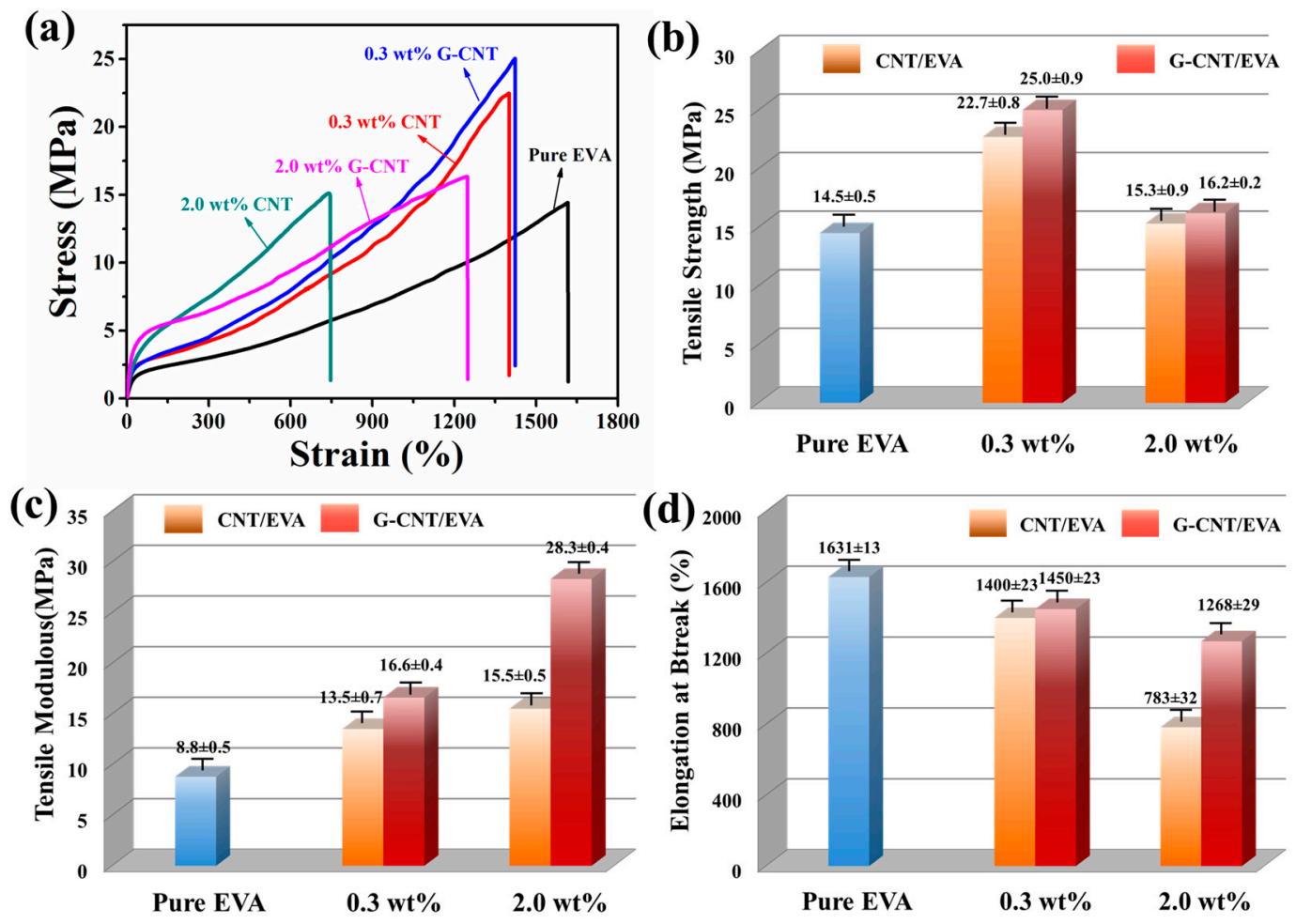

Figure 4. (a) Typical stress-strain curves of pure ethylene vinyl acetate (EVA) and its composites with various CNT loadings; (b) Tensile strength, (c) Young's modulus, and (d) elongation at break of pure EVA and its composites.

\subsection{Effects of CNT Dispersion on Electrical Properties}

Figure 5 shows the electrical conductivities of the G-CNT/EVA and CNT/EVA composites as a function of CNT content. Significant increases in the electrical conductivities of both the G-CNT/EVA and the CNT/EVA composites are observed with increasing CNT contents. Interestingly, the G-CNT/EVA composites exhibit superior electrical conductivities at low CNT contents, but inferior electrical conductivities at high CNT contents, compared to the CNT/EVA composites. At a very low CNT content of $0.2 \mathrm{wt} \%$, the electrical conductivity of G-CNT/EVA composite already reaches $6.9 \times 10^{-8} \mathrm{~S} / \mathrm{m}$, satisfying the antistatic criterion for commercial application [44]. Such value is about 4 orders of magnitude higher than that of the CNT/EVA composite $\left(4.3 \times 10^{-12} \mathrm{~S} / \mathrm{m}\right)$. As the CNT loading rises to $0.5 \mathrm{wt} \%$, the electrical conductivity of the G-CNT/EVA composites increases to $4.9 \times 10^{-6} \mathrm{~S} / \mathrm{m}$, which is still higher than that of CNT/EVA composites. However, with further increasing CNT loadings to $1.0 \mathrm{wt} \%$ and $2.0 \mathrm{wt} \%$, it can be seen that the electrical conductivities of CNT/EVA composites exceed that of the G-CNT/EVA composites. The main reasons for the interesting phenomenon can be found in the following two aspects. The first aspect is that the incorporation of GO-ODA can significantly improve CNT dispersion in EVA matrix due to $\pi-\pi$ stacking interaction between GO-ODA and CNT. This contributes to the formation of inter-connected CNT networks. The second aspect is that the abundant presence of insulating GO-ODA between the adjacent CNTs will increase their contact resistance and thus hinder the electronic transport of CNTs in EVA. To provide visual demonstration of the conductive networks in the G-CNT/EVA and CNT/EVA composites, schematic representations are shown in Figure $5 b, c$ and Figure 5 d,e, respectively. At low CNT loading, the improved CNT dispersion contributes to the formation of effective conductive networks in the G-CNT/EVA composites (Figure 5b), while conductive networks are not formed in the CNT/EVA composites due to the agglomeration of CNTs (Figure 5d). Thus the first aspect plays a leading role in the electrical conductivity of the composites, which impart the G-CNT/EVA composites with higher electrical conductivity than that of the CNT/EVA composites. At high CNT loading, the CNT amount 
is enough to form inter-connected CNT networks in both the G-CNT/EVA composites (Figure 5c) and CNT/EVA composites (Figure 5e). The second aspect is dominant for the electrical conductivity of the composites and thus the G-CNT/EVA composites exhibit inferior electrical conductivity compared to that of CNT/EVA composites. A similar phenomenon was also reported for other CNT-based composites with the assistance of surfactants to improve CNT dispersion [45].
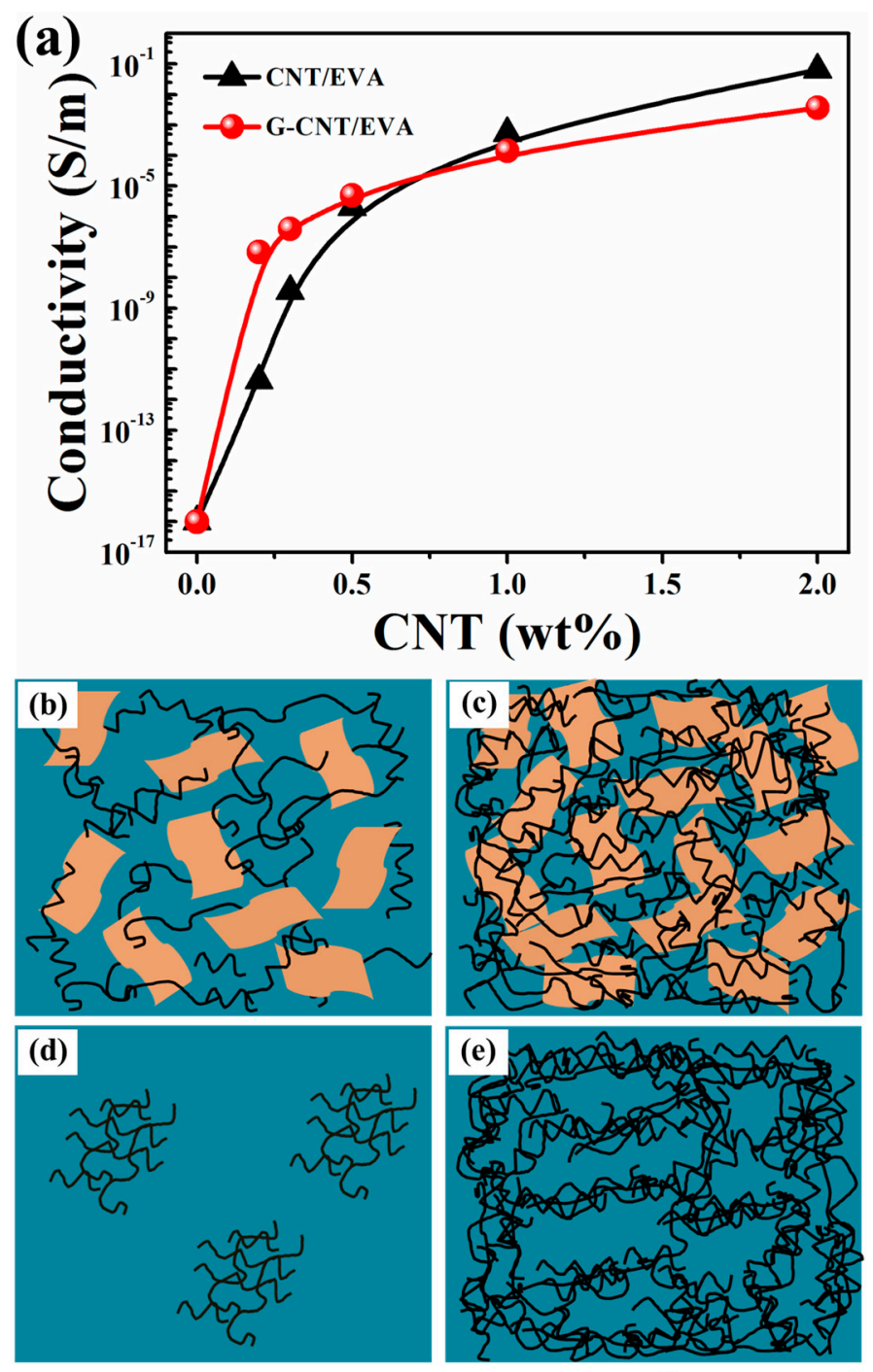

Increasing CNT content

Figure 5. (a) Electrical conductivities of the G-CNT/EVA and the CNT/EVA composites. (b) and (c) The scheme of the conductive networks in the G-CNT/EVA composites at low and high CNT contents, respectively. (d) and (e) The scheme of the conductive networks in the CNT/EVA composites at low and high CNT contents, respectively.

\section{Conclusions}

We demonstrate a facile and effective approach to realize the homogeneous dispersion of CNT in EVA matrix, by using GO-ODA as a compatilizer. The resultant G-CNT/EVA composites show superior mechanical properties in comparison with the CNT/EVA composites, which could be attributed to the uniform dispersion of CNT in EVA matrix and the strong interfacial interaction between CNT and EVA, originating from the solubilization effect of GO-ODA. At a very low CNT content of $0.2 \mathrm{wt} \%$, the electrical conductivity of G-CNT/EVA already reaches $6.9 \times 10^{-8} \mathrm{~S} / \mathrm{m}$, satisfying the antistatic criterion for commercial application. The concept of dispersing CNT by using 
hydrophobic GO-ODA in our work can be easily extended to other CNT-based polymer systems, which paves the way to develop such composites with excellent properties.

Acknowledgments: The authors gratefully acknowledge the financial support from the National Natural Science Foundation of China (Grant No. 51673134, 51421061, and 51473102,), the Science and Technology Department of Sichuan Province (Grant No. 2017GZ0412).

Author Contributions: Ding-Xiang Yan and Zhong-Ming Li conceived and designed the experiments; Li-Chuan Jia and Zhong-Han Jiao performed the experiments; Li-Chuan Jia, Ding-Xiang Yan and Zhong-Ming Li analyzed the data; Li-Chuan Jia wrote the paper; the paper was reviewed by all authors.

Conflicts of Interest: The authors declare no conflict of interest.

\section{References}

1. Chen, H.; Zeng, S.; Chen, M.; Zhang, Y.; Li, Q. Fabrication and functionalization of carbon nanotube films for high-performance flexible supercapacitors. Carbon 2015, 92, 271-296. [CrossRef]

2. Zhang, W.B.; Xu, X.L.; Yang, J.H.; Huang, T.; Zhang, N.; Wang, Y.; Zhou, Z.W. High thermal conductivity of poly (vinylidene fluoride)/carbon nanotubes nanocomposites achieved by adding polyvinyl pyrrolidone. Compos. Sci. Technol. 2015, 106, 1-8. [CrossRef]

3. Jia, L.C.; Yan, D.X.; Yang, Y.; Zhou, D.; Cui, C.H.; Bianco, E.; Lou, J.; Vajtai, R.; Li, B.; Ajayan, P.M.; et al. High strain tolerant emi shielding using carbon nanotube network stabilized rubber composite. Adv. Mater. Technol. 2017, 2, 1700078. [CrossRef]

4. Hayashida, K.; Tanaka, H. Ultrahigh electrical resistance of poly(cyclohexyl methacrylate)/carbon nanotube composites prepared using surface-initiated polymerization. Adv. Funct. Mater. 2012, 22, 2338-2344. [CrossRef]

5. Jia, L.C.; Yan, D.X.; Cui, C.H.; Jiang, X.; Ji, X.; Li, Z.M. Electrically conductive and electromagnetic interference shielding of polyethylene composites with devisable carbon nanotube networks. J. Mater. Chem. C 2015, 3, 9369-9378. [CrossRef]

6. Huang, J.; Mao, C.; Zhu, Y.; Jiang, W.; Yang, X. Control of carbon nanotubes at the interface of a co-continuous immiscible polymer blend to fabricate conductive composites with ultralow percolation thresholds. Carbon 2014, 73, 267-274. [CrossRef]

7. George, N.; Chandra, C.S.J.; Mathiazhagan, A.; Joseph, R. High performance natural rubber composites with conductive segregated network of multiwalled carbon nanotubes. Compos. Sci. Technol. 2015, 116, 33-40. [CrossRef]

8. De Volder, M.F.; Tawfick, S.H.; Baughman, R.H.; Hart, A.J. Carbon nanotubes: present and future commercial applications. Science 2013, 339, 535-539. [CrossRef] [PubMed]

9. Jia, L.C.; Li, M.Z.; Yan, D.X.; Cui, C.H.; Wu, H.-Y.; Li, Z.M. A strong and tough polymer-carbon nanotube film for flexible and efficient electromagnetic interference shielding. J. Mater. Chem. C 2017. [CrossRef]

10. Zhou, J.; Yu, H.; Xu, X.; Han, F.; Lubineau, G. Ultrasensitive, stretchable strain sensors based on fragmented carbon nanotube papers. ACS Appl. Mater. Interfaces 2017, 9, 4835-4842. [CrossRef] [PubMed]

11. Cui, C.H.; Yan, D.X.; Pang, H.; Jia, L.C.; Xu, X.; Yang, S.; Xu, J.Z.; Li, Z.M. A high heat-resistance bioplastic foam with efficient electromagnetic interference shielding. Chem. Eng. J. 2017, 323, 29-36. [CrossRef]

12. Li, Y.; Zhang, Z.; Li, X.; Zhang, J.; Lou, H.; Shi, X.; Cheng, X.; Peng, H. A smart, stretchable resistive heater textile. J. Mater. Chem. C 2017, 5, 41-46. [CrossRef]

13. Jia, L.C.; Li, Y.K.; Yan, D.X. Flexible and efficient electromagnetic interference shielding materials from ground tire rubber. Carbon 2017, 121, 267-273. [CrossRef]

14. Chen, Y.; Zhang, H.B.; Yang, Y.; Wang, M.; Cao, A.; Yu, Z.Z. High-performance epoxy nanocomposites reinforced with three-dimensional carbon nanotube sponge for electromagnetic interference shielding. Adv. Funct. Mater. 2016, 26, 447-455. [CrossRef]

15. Zhang, K.; Yu, H.O.; Shi, Y.D.; Chen, Y.F.; Zeng, J.B.; Guo, J.; Wang, B.; Guo, Z.; Wang, M. Morphological regulation improved electrical conductivity and electromagnetic interference shielding in poly (L-lactide)/poly ( $\xi$-caprolactone)/carbon nanotubes nanocomposites via constructing stereocomplex crystallites. J. Mater. Chem. C 2017, 5, 2807-2817. [CrossRef] 
16. Atar, N.; Grossman, E.; Gouzman, I.; Bolker, A.; Hanein, Y. Reinforced carbon nanotubes as electrically conducting and flexible films for space applications. ACS Appl. Mater. Interfaces 2014, 6, 20400-20407. [CrossRef] [PubMed]

17. Han, J.H.; Zhang, H.; Chen, M.J.; Wang, G.R.; Zhang, Z. CNT buckypaper/thermoplastic polyurethane composites with enhanced stiffness, strength and toughness. Compos. Sci. Technol. 2014, 103, 63-71. [CrossRef]

18. Oh, J.Y.; Jun, G.H.; Jin, S.; Ryu, H.J.; Hong, S.H. Enhanced electrical networks of stretchable conductors with small fraction of carbon nanotube/graphene hybrid fillers. ACS Appl. Mater. Interfaces 2016, 8, 3319-3325. [CrossRef] [PubMed]

19. Souier, T.; Maragliano, C.; Stefancich, M.; Chiesa, M. How to achieve high electrical conductivity in aligned carbon nanotube polymer composites. Carbon 2013, 64, 150-157. [CrossRef]

20. Niyogi, S.; Hamon, M.A.; Hu, H.; Zhao, B.; Bhowmik, P.; Sen, R.; Itkis, M.E.; Haddon, R.C. Chemistry of single-walled carbon nanotubes. Accounts Chem. Res. 2002, 35, 1105-1113. [CrossRef]

21. Hu, L.; Hecht, D.S.; Gruner, G. Carbon nanotube thin films: fabrication, properties, and applications. Chem. Rev. 2010, 110, 5790-5844. [CrossRef] [PubMed]

22. Choi, E.Y.; Roh, S.C.; Kim, C.K. Noncovalent functionalization of multi-walled carbon nanotubes with pyrene-linked nylon66 for high performance nylon66/multi-walled carbon nanotube composites. Carbon 2014, 72, 160-168. [CrossRef]

23. Roy, S.; Das, T.; Yue, C.Y.; Hu, X. Improved polymer encapsulation on multiwalled carbon nanotubes by selective plasma induced controlled polymer grafting. ACS Appl. Mater. Interfaces 2014, 6, 664-670. [CrossRef] [PubMed]

24. Daugaard, A.E.; Jankova, K.; Hvilsted, S. Poly(lauryl acrylate) and poly(stearyl acrylate) grafted multiwalled carbon nanotubes for polypropylene composites. Polymer 2014, 55, 481-487. [CrossRef]

25. George, N.; Bipinbal, P.K.; Bhadran, B.; Mathiazhagan, A.; Joseph, R. Segregated network formation of multiwalled carbon nanotubes in natural rubber through surfactant assisted latex compounding: A novel technique for multifunctional properties. Polymer 2017, 112, 264-277. [CrossRef]

26. Byrne, M.T.; Gun'ko, Y.K. Recent advances in research on carbon nanotube-polymer composites. Adv. Mater. 2010, 22, 1672-1688. [CrossRef] [PubMed]

27. Zhang, C.; Huang, S.; Tjiu, W.W.; Fan, W.; Liu, T. Facile preparation of water-dispersible graphene sheets stabilized by acid-treated multi-walled carbon nanotubes and their poly (vinyl alcohol) composites. J. Mater. Chem. 2012, 22, 2427-2434. [CrossRef]

28. Zhang, C.; Ren, L.; Wang, X.; Liu, T. Graphene oxide-assisted dispersion of pristine multiwalled carbon nanotubes in aqueous media. J. Phys. Chem. C 2010, 114, 11435-11440. [CrossRef]

29. Tian, L.; Meziani, M.J.; Lu, F.; Kong, C.Y.; Cao, L.; Thorne, T.J.; Sun, Y.P. Graphene oxides for homogeneous dispersion of carbon nanotubes. ACS Appl. Mater. Interfaces 2010, 2, 3217-3222. [CrossRef] [PubMed]

30. Dong, X.; Xing, G.; Chan-Park, M.B.; Shi, W.; Xiao, N.; Wang, J.; Yan, Q.; Sum, T.C.; Huang, W.; Chen, P. The formation of a carbon nanotube-graphene oxide core-shell structure and its possible applications. Carbon 2011, 49, 5071-5078. [CrossRef]

31. Li, Y.; Yang, T.; Yu, T.; Zheng, L.; Liao, K. Synergistic effect of hybrid carbon nantube-graphene oxide as a nanofiller in enhancing the mechanical properties of PVA composites. J. Mater. Chem. 2011, 21, 10844-10851. [CrossRef]

32. Shen, X.J.; Pei, X.Q.; Liu, Y.; Fu, S.Y. Tribological performance of carbon nanotube-graphene oxide hybrid/epoxy composites. Compos. B 2014, 57, 120-125. [CrossRef]

33. Wu, C.; Huang, X.; Wu, X.; Xie, L.; Yang, K.; Jiang, P. Graphene oxide-encapsulated carbon nanotube hybrids for high dielectric performance nanocomposites with enhanced energy storage density. Nanoscale 2013, 5, 3847-3855. [CrossRef] [PubMed]

34. Choudhary, S.; Mungse, H.P.; Khatri, O.P. Dispersion of alkylated graphene in organic solvents and its potential for lubrication applications. J. Mater. Chem. 2012, 22, 21032-21039. [CrossRef]

35. Ryu, S.H.; Shanmugharaj, A. Influence of long-chain alkylamine-modified graphene oxide on the crystallization, mechanical and electrical properties of isotactic polypropylene nanocomposites. Chem. Eng. J. 2014, 244, 552-560. [CrossRef] 
36. Huang, H.D.; Zhou, S.Y.; Ren, P.G.; Ji, X.; Li, Z.M. Improved mechanical and barrier properties of low-density polyethylene nanocomposite films by incorporating hydrophobic graphene oxide nanosheets. RSC Adv. 2015, 5, 80739-80748. [CrossRef]

37. Jia, L.C.; Yan, D.X.; Cui, C.H.; Ji, X.; Li, Z.M. A unique double percolated polymer composite for highly efficient electromagnetic interference shielding. Macromol. Mater. Eng. 2016, 301, 1232-1241. [CrossRef]

38. Clancy, T.C.; Gates, T.S. Modeling of interfacial modification effects on thermal conductivity of carbon nanotube composites. Polymer 2006, 47, 5990-5996. [CrossRef]

39. Zhang, Z.; Zhang, Y.; Yang, K.; Yi, K.; Zhou, Z.; Huang, A.; Mai, K.; Lu, X. Three-dimensional carbon nanotube/ethylvinylacetate/polyaniline as a high performance electrode for supercapacitors. J. Mater. Chem. A 2015, 3, 1884-1889. [CrossRef]

40. Yan, D.X.; Pang, H.; Li, B.; Vajtai, R.; Xu, L.; Ren, P.G.; Wang, J.H.; Li, Z.M. Structured reduced graphene oxide/polymer composites for ultra-efficient electromagnetic interference shielding. Adv. Funct. Mater. 2015, 25, 559-566. [CrossRef]

41. Li, W.; Tang, X.Z.; Zhang, H.B.; Jiang, Z.G.; Yu, Z.Z.; Du, X.S.; Mai, Y.W. Simultaneous surface functionalization and reduction of graphene oxide with octadecylamine for electrically conductive polystyrene composites. Carbon 2011, 49, 4724-4730. [CrossRef]

42. Lin, Z.; Liu, Y.; Wong, C.P. Facile fabrication of superhydrophobic octadecylamine-functionalized graphite oxide film. Langmuir 2010, 26, 16110-16114. [CrossRef]

43. Yao, H.; Chu, C.C.; Sue, H.J.; Nishimura, R. Electrically conductive superhydrophobic octadecylamine-functionalized multiwall carbon nanotube films. Carbon 2013, 53, 366-373. [CrossRef]

44. Verma, M.; Verma, P.; Dhawan, S.; Choudhary, V. Tailored graphene based polyurethane composites for efficient electrostatic dissipation and electromagnetic interference shielding applications. RSC Adv. 2015, 5, 97349-97358. [CrossRef]

45. Kim, S.W.; Kim, T.; Kim, Y.S.; Choi, H.S.; Lim, H.J.; Yang, S.J.; Park, C.R. Surface modifications for the effective dispersion of carbon nanotubes in solvents and polymers. Carbon 2012, 50, 3-33. [CrossRef]

(C) 2017 by the authors. Licensee MDPI, Basel, Switzerland. This article is an open access article distributed under the terms and conditions of the Creative Commons Attribution (CC BY) license (http:/ / creativecommons.org/licenses/by/4.0/). 